\title{
NONLINEAR INTERACTION OF WAVES IN A HOT INHOMOGENEOUS MAGNETIZED PLASMA
}

\author{
TARA PRASAD KHAN \\ Physics Department \\ Dinabandhu Andrews College \\ Dist - 24 Parganas, West Bengal, India
}

MAHADEB DAS

Physics Department

Serampore College, Serampore

Dist - Hooghly, West Bengal, India

\section{LOKENATH DEBNATH}

Departments of Mathematics and Physics

East Carolina University

Greenville, N.C. 27834, U.S.A.

(Received August 7, 1978 and in revised form May 1, 1979)

ABSTRACT. This study is concerned with the theory of parametric coupling of waves in a hot inhomogeneous magnetized plasma in which the temperature gradient has been taken into account. The general dispersion relation and the polarization of the ordinary and the extra-ordinary wave modes are discussed. The eigen-mode solutions of the coupled differential equations for the wave amplitudes are obtained in the terms of the so called three wave interaction matrix elements. The theory of nonlinear wave-wave interactions, which has been extended to the case of an inhomogeneous magnetized plasma, is used to determine the threshold value of the electric field and the frequency shift. The results of this paper are also compared 
with the other known results. It is shown that the findings of this study are in excellent agreement with the results of earlier investigators.

KEY WORDS AND PHRASES. Parametric instability, waves in inhomogeneous plasma, wave-wave interactions. Threshold electric field and frequency shift.

\section{MATHEMATICS SUBJECT CLASSIFICATION CODES. 76X05, $76 E 30$.}

\section{INTRODUCTION.}

In recent years there has been considerable interest in the theory of parametricinstability in an inhomogeneous plasma [1-8] because of its fundamental role in the study of weak plasma turbulence, and of many important physical applications. In their recent research-expository and survey article on parametric phenomena in a plasma, Galeev and Sagdeev [9] reviewed the theory of parametric instabilities in an inhomogeneous plasma using the generalized Mathieu equation as a model equation. They have also presented the latest advances of the nonlinear theories of parametric instabilities based on the ideas of weakly turbulent plasma. Perkins and Flick [10] have made an interesting study of parametric instabilities in an inhomogeneous plasmas, and then calculated the threshold electric field. It is shown that the value of the threshold electric field increases in an inhomogeneous plasma because energy propagates away from the unstable region by electron plasma waves. Thus an additional energy loss occurs and is solely responsible for the increase of the threshold electric field not observed in a homogeneous plasma. Eubank [11] experimentally confirmed the theoretical prediction of Perkins and Flick. In a recent paper, Kroll, Ron and Rostoker [12] have suggested that the nonlinear resonance of two transverse electromagnetic waves whose frequencies differ slightly by the electron plasma frequency can be applied to excite longitudinal electron plasma oscillations. Montgomery [13] observed certain mathematical inaccuracy and physical limitations of the work of Kroll, Ron and Rostoker, and then analyzed the problem of nonlinear wave interactions in plasma with laser beams. 
Using the perturbation method of Krylov-Bogliubov-Mitropolskii, Montgomery obtained the amplitude-dependent frequency shift and wave number shift with physical significance. It was shown that the resonant excitation of longitudinal plasma oscillations is possible by the transverse electromagnetic waves. Etievant, Ossakow, Ozizmir and $\mathrm{Su}[14]$ have investigated the nonlinear wave-wave interactions of electromagnetic waves in an infinite homogeneous plasma. It is interesting and important to take into account the effects of density and temperature gradients on the above problem.

The present study deals with the theory of parametric coupling of waves in a hot inhomogeneous magnetized plasma in which the temperature gradient has been taken into consideration. The general dispersion relation and the polarization of two different wave modes are investigated. The eigen-mode solutions of the coupled equations for the wave amplitudes are obtained in terms of the three wave interaction matrix elements. The theory of nonlinear wave-wave interactions is used to determine the threshold electric field and the frequency shift. The results of this analysis are found to be in excellent agreement with those of earlier workers.

\section{BASIC EQUATIONS FOR TWO PLASMA MODEL.}

In two plasma model, the equations of motion and the continuity equation for each kind of component are in the usual notations given by

$$
\begin{gathered}
\frac{\mathrm{D} \underline{\mathrm{v}}_{\alpha}}{\mathrm{Dt}}=\frac{\mathrm{Z}_{\alpha} \mathrm{e}}{\mathrm{m}_{\alpha}} \quad \underline{\mathrm{E}}-\frac{\mathrm{K}}{\mathrm{m}_{\alpha}} \frac{\nabla\left(\mathrm{T}_{\alpha} \rho_{\alpha}\right)}{\rho_{\alpha}}-\mathrm{z}_{\alpha} \Omega_{\alpha} \times \underline{\mathrm{v}}_{\alpha}-\nu_{\alpha} \mathrm{v}_{\alpha}, \\
\frac{\mathrm{D} \rho_{\alpha}}{\mathrm{Dt}}+\rho_{\alpha} \nabla \cdot \underline{\mathrm{v}}_{\bar{\alpha}}=0,
\end{gathered}
$$

where $\Omega_{\alpha}=\frac{\mathrm{eB}_{0}}{\mathrm{~cm}_{\alpha}}$ is the cyclotron frequency, $\nu_{\alpha}$ is the collision frequency of each 
component and $B=B_{0} \hat{z}$ is the external magnetic field. The subscript $\alpha$ stands for e or 1 corresponding to electrons and ions respectively, $z_{\alpha} e$ is the charge of an electron or ion and $k$ is the Boltzmann Constant.

The Maxwe11 equations are

$$
\begin{aligned}
& \operatorname{cur1} \underline{E}=-\frac{1}{c} \frac{\partial \underline{B}}{\partial t}, \quad \operatorname{div} \underline{E}=-4 \pi e\left(n-n_{0}\right), \\
& \operatorname{cur1} \underline{B}=\frac{1}{c} \frac{\partial \underline{E}}{\partial t}-\frac{4 \pi}{c} \operatorname{nev}_{\alpha}, \quad \operatorname{div} \underline{B}=0,
\end{aligned}
$$

We consider a plasma model whose density distribution varies as $\rho_{\alpha}=\rho_{\alpha 0}(1-x \delta)$ with the density gradient $\delta=\frac{1}{\rho_{\alpha O}}\left(\frac{\mathrm{d} \rho}{\mathrm{dx}}\right)$ and neglect the time dependence of $\rho_{\alpha \circ}$ due to any external electric field. The zeroeth order solution of (2.1) gives

$$
\begin{aligned}
& \left\langle v_{o x}(t)\right\rangle_{e}=\frac{k T_{e}}{m_{e}} \frac{\delta \nu_{e}}{\left(\Omega_{e}^{2}-\nu_{e}^{2}\right)} \text {, } \\
& \left\langle v_{\text {oy }}(t)\right\rangle_{e}=\frac{k \mathrm{~T} e}{m_{e}} \frac{\delta \Omega_{e} Z_{e}}{\left(\Omega_{e}{ }^{2}-\nu_{e}{ }^{2}\right)}, \\
& \underline{\delta}=\delta \hat{\mathbf{x}}=\left(\frac{\nabla \rho_{0}}{\rho_{0}}+\frac{\nabla \mathrm{T}_{0}}{\mathrm{~T}_{0}}\right)
\end{aligned}
$$

\section{THE FIRST ORDER EQUATIONS.}

In the first order approximation, equations (2.1) - (2.2) can be written as, dropping the subscript $\alpha$,

$$
\frac{\partial \underline{v}_{1}}{\partial t}+\underline{v}_{0} \cdot \nabla v_{1}+\underline{v}_{1} \cdot \nabla v_{0}=\frac{Z e}{m} \underline{E}_{0}-\underline{z}_{0} \times \underline{v}_{1}-\frac{k}{m} \frac{\nabla(T \rho)}{\rho}-\underline{Z}_{-1} \times \underline{v}_{0}-\underline{v}_{-1},
$$

where $\left(\frac{\nabla T \rho}{\rho}\right)_{1}$ represents the first order terms in $\left(\frac{\nabla T \rho}{\rho}\right)$. 
Assuming the density gradient is small, we take the space and time dependent of the first order quantities as $\exp [i(\underline{k} \cdot \underline{r}-\omega t)]$. The equation (3.1) can then readily be solved to obtain

$$
\underline{\mathrm{v}}_{1}=\underline{\mu} \cdot \underline{\mathrm{E}}_{1},
$$

where $\underline{\mu}$ is the mobility tensor given by

$$
\underline{\mu}_{\alpha}=\frac{z_{e}}{m_{a}} \underline{\mu}_{c} \cdot\left[\left(1-\frac{\underline{k} \cdot \underline{v}_{0}}{\omega}\right) I+\frac{\underline{k} \cdot \underline{v}_{0}}{\omega}\right] \text {, }
$$

when $I$ is the unit dyadic and $\underline{\mu}_{c}$ is

$$
\left.\underline{\mu}_{c}=\frac{1}{\Delta}\left[\overline{(\bar{\omega}}-\underline{k} \cdot \underline{v}_{0}\right)(\hat{x x}+\hat{y} \hat{y})-\tilde{G y y}\right] \pm \frac{\Delta \hat{z} \hat{z}}{\left(\bar{\omega}-\underline{k} \cdot \underline{v}_{0}^{\prime}\right)}+i z \Omega_{0} \hat{x y}-i z \Omega_{0} \hat{y x},
$$

with

$$
\begin{aligned}
& \tilde{G}=\frac{k T}{m\left(\bar{\omega}-\underline{k} \cdot \underline{v}_{0}\right)}\left\{\gamma k^{2}+2 i(1-\gamma) k \delta\right\}, \\
& \Delta=\left(\bar{\omega}-\underline{k} \cdot \underline{v}_{0}\right)^{2}-\left(\bar{\omega}-\underline{k} \cdot \underline{v}_{0}\right) \tilde{G}-\Omega_{0}{ }^{2}, \\
& \bar{\omega}=\omega+i \nu
\end{aligned}
$$

and $\gamma$ is the ratio of the two specific heats.

Using equations (3.2) and taking the Maxwell equations (2.3ab) - (2.4ab), it turns out that

$$
D \cdot \underline{E}_{1}=0,
$$

with

$$
D=\left(k^{2}-\frac{\omega^{2}}{c^{2}}\right) I-\hat{k} \hat{k}-\frac{i \omega}{c^{2}} \sum \omega_{\alpha}^{2}\left(I+\frac{\underline{y}_{\alpha 0} \underline{k}}{\omega}\right) \cdot \mu_{c},
$$

where

$$
\omega_{\alpha}^{2}=\frac{4 \pi e \rho_{\alpha}}{m_{a}} \text {, }
$$

In the case of wave propagation along with $x$-direction $(\underline{k}=\hat{k x})$, we obtain 
the dispersion relation

$$
\text { Det } D(k, \omega)=o \text {, }
$$

There are two independent solutions of equation (3.10) in the form

$$
\mathrm{D}_{\mathrm{zz}}=0,
$$

which corresponds to the ordinary wave, and

$$
D_{x x} D_{y y}-D_{x y} D_{y x}=0
$$

which corresponds to the extra-ordinary wave, where $D_{x x}, D_{y y}, \ldots \ldots \ldots$ are the elements of the matrix $D$.

The first order fields for the different modes (ordinary and extra-ordinary) are obtained as

$$
\begin{aligned}
\underline{E}_{0} & =\hat{a}_{0} A_{0} \exp \left[i\left(\underline{k}_{0} \cdot \underline{r}-\omega_{0} t\right)\right], \\
\underline{E}_{e} & =\hat{a}_{e} A_{e} \exp \left[i\left(\underline{k}_{e} \cdot \underline{r}-\omega_{e} t\right)\right], \\
\text { with } \quad a_{0} & =\hat{z}, a_{e}=(i b \hat{x}+\hat{y}) /\left(1+b^{2}\right)^{\frac{1}{2}},
\end{aligned}
$$$$
\text { and } \quad b=-i \frac{E_{x}}{E_{y}}=\frac{i D_{y y}}{D_{y x}} \text {, }
$$

where

$\mathrm{D}_{\mathrm{yy}}=\left(\mathrm{k}^{2}-\frac{\omega^{2}}{\mathrm{c}^{2}}\right)+\sum_{\alpha} \frac{\omega_{\alpha}{ }^{2} \omega}{\mathrm{c}^{2} \Delta}\left[\left(\bar{\omega}-\underline{\mathrm{k}} \cdot \underline{\mathrm{v}}_{\mathrm{o}}-\tilde{\mathrm{G}}\right)+\frac{i z \Omega_{0} \mathrm{kv}_{\mathrm{oy}}}{\omega}\right]$

$\mathrm{D}_{\mathrm{yx}}=\sum_{\alpha} \frac{\omega \omega_{\alpha}^{2}}{c^{2}}\left[\frac{\left(\bar{\omega}-\underline{\mathrm{k}} \cdot \underline{\mathrm{v}}_{0}\right) \mathrm{k} \mathrm{v}_{\mathrm{oy}}}{\omega \Delta}-\mathrm{z} \Omega_{0}\right]$

4. THE SECOND ORDER EQUATIONS.

In the second order approximation, we obtain from equations (2.1) - (2.2) 


$$
\begin{aligned}
\frac{\partial \underline{v}_{2}}{\partial t}+\underline{v}_{0} \cdot \nabla v_{2}+\underline{v}_{1} \cdot \nabla v_{1}+\underline{v}_{2} \cdot \nabla v_{0} & =\frac{z e}{m} \underline{\mathrm{E}}_{2}-\underline{v}_{2}-\frac{z \Omega}{0} \times \underline{v}_{2}-z \underline{\Omega}_{1} \times \underline{v}_{1} \\
& -\frac{z \Omega_{2}}{\underline{v}_{0}} \underline{v}_{0}+\frac{\kappa}{m}\left(\frac{\nabla T \rho}{\rho}\right)_{2},
\end{aligned}
$$

with

$\frac{k}{\mathrm{~m}}\left(\frac{\nabla \mathrm{T} \rho}{\rho}\right)_{2}=\frac{i k \mathrm{~T}}{\mathrm{~m}}\left[\{\underline{\underline{k}}-i(\gamma-2) \underline{\delta}\} \frac{\rho_{2}}{\rho_{0}}+\frac{\gamma-1}{2}\{\underline{\gamma} \underline{\mathrm{k}}-i(\gamma-2) \underline{\delta}\} \frac{\rho_{1}{ }^{2}}{\rho_{0}{ }^{2}}+\frac{i \gamma \rho_{1} \nabla \rho_{1}}{\rho_{0}{ }^{2}}\right]$

$\frac{\rho_{z}}{\rho_{0}}=\left[\frac{\rho_{0}}{\rho_{1}}\left(\underline{\mathrm{k}} \cdot \underline{\mathrm{v}}_{1}\right)+(\underline{\mathrm{k}}-\mathbf{i} \underline{\delta}) \cdot \underline{\mathrm{v}}_{2}\right] \div\left(\omega-\underline{\mathrm{k}} \cdot \underline{\mathrm{v}}_{0}\right)$,

Using the space and time dependence of all second order quantities in the form $\exp [i(\underline{k} \cdot \underline{r}-\omega t)]$, we find

$$
\underline{\mathrm{v}}_{2}=\frac{\mathrm{Ze}}{\mathrm{m}} \mu_{\mathrm{c}} \cdot\left[\left(1-\frac{\underline{\mathrm{k}} \cdot \underline{\mathrm{v}}_{0}}{\omega}\right) \mathrm{I}+\frac{\underline{\mathrm{k}} \cdot \underline{\mathrm{v}}_{\mathrm{O}}}{\omega}\right] \cdot \underline{\mathbf{E}}_{2}+\mu_{\mathrm{c}} \cdot \underline{\mathrm{H}},
$$

with

$$
\underline{\mathrm{H}}=-\underline{\mathrm{v}}_{1} \cdot \nabla \mathrm{v}_{1}-\underline{\Omega}_{1} \times \underline{\mathrm{v}}_{1}-\mathrm{i} \mathrm{Q} \text {, }
$$

$$
\begin{aligned}
& Q=\frac{k T}{m}\left[\frac{\rho_{1}\left(\underline{k} \cdot \underline{v}_{1}\right)(\gamma \underline{k}-i(\gamma-2) \underline{\delta}}{\rho_{0}\left(\omega-\underline{k} \cdot \underline{v}_{0}\right)}+\frac{\gamma-1}{2}\{\gamma \underline{k}-i(\gamma-2) \underline{\delta}\} \frac{\rho_{1}{ }^{2}}{\rho_{0}{ }^{2}}\right. \\
& \left.+i \gamma \rho_{1} \frac{\nabla \rho_{1}}{\rho_{0}^{2}}\right]
\end{aligned}
$$

Using the Maxwell equations and eliminating the second order magnetic field $B_{2}$, it follows that

$$
\nabla \times\left(\nabla \times \underline{E}_{2}\right)=\frac{1}{c^{2}} \frac{\partial^{2} E_{2}}{\partial t^{2}}=-\frac{4 \pi}{c^{2}} \frac{\partial \underline{J}_{2}}{\partial t},
$$

whence

$$
\underline{\mathrm{J}}_{2}=\rho_{\mathrm{o}} \underline{\mathrm{v}}_{2}+\rho_{2} \mathrm{v}_{0}+\rho_{1} \underline{\mathrm{v}}_{1} \text {, }
$$

Substituting the values of $\underline{v}_{2}$ in equation (4.4), we obtain

$$
\begin{aligned}
& \nabla \times\left(\nabla \times \underline{E}_{2}\right)+\frac{1}{c^{2}} \frac{\partial^{2} E_{2}}{\partial t^{2}}+\frac{4 \pi}{c^{2}} \sum_{\alpha}\left(\rho_{\alpha 0} \mu_{\alpha}+\frac{\rho_{\alpha O} \underline{v}_{\alpha O} \underline{k} \cdot \mu_{\alpha}}{\omega-\underline{k} \cdot \underline{v}_{\alpha o}}\right) \frac{\partial E_{2}}{\partial t} \\
& =\frac{4 \pi i \omega}{c^{2}} \sum_{\alpha} \rho_{\alpha o}\left(I+\frac{\underline{v}_{\alpha O} \underline{k}}{\omega}\right) \cdot\left(\mu_{c} \cdot \underline{H}_{\alpha}+\frac{\underline{v}_{\alpha 1} \rho_{\alpha 1}}{\rho_{\alpha o}}\right),
\end{aligned}
$$




\section{EQUATION FOR THE INTERACTING MODES.}

We assume a plane wave solution with an amplitude varying in the direction of propagation and write

$$
\underline{E}_{2}=\hat{a}_{\omega} A_{\omega}(x, t) \quad \exp [i(\underline{k} \cdot \underline{r}-\omega t)],
$$

where $A_{\omega}$ is a slowly varying function of $x$ and $t$ because the nonlinearity is assumed to be weak.

We next drop the second derivatives of $\mathrm{A}$ in comparison to $\mathrm{k}^{2} \mathrm{~A}$ and $\omega^{2} \mathrm{~A}$, and then use the linear dispersion relation $D \cdot \underline{E}_{2}=0$ to obtain the final result $\left(u_{1} \frac{\partial}{\partial x}+\frac{\partial}{\partial t}\right) A_{1}=A_{3} A_{2} v_{123}\left(\omega_{1}\left|\omega_{2}\right| \omega_{3}\right) \exp \{i(x \Delta \underline{k}-t \Delta \omega)\}$,

$\left(u_{2} \frac{\partial}{\partial x}+\frac{\partial}{\partial t}\right) A_{2}=A_{1} A_{3} v_{213}\left(-\omega_{2}\left|-\omega_{1}\right| \omega_{3}\right) \exp \{i(-x \Delta \underline{k}+t \Delta \omega)\}$,

$\left(u_{3} \cdot \frac{\partial}{\partial x}+\frac{\partial}{\partial t}\right) A_{3}=A_{1} A_{2} V_{321}\left(-\omega_{3}\left|\omega_{2}\right|-\omega_{1}\right) \exp \{i(-x \Delta \underline{k}+t \Delta \omega)\}$,

where $\underline{\Delta k}=\underline{k}_{3}-\left(\underline{k}_{2}+\underline{k}_{1}\right)$ and $\Delta \omega=\omega_{3}-\left(\omega_{1}+\omega_{2}\right)$,

We write the matrix elements for $\hat{a}_{1}=\hat{a}_{3}=a_{0}$ and $\hat{a}_{2}=a_{e}$ where $a_{o}$ and $a_{e}$ are defined in (3.15ab).

$$
\begin{aligned}
& v_{123}=2 \pi i \sum_{\alpha} \rho_{\alpha O}\left\{-i\left(\hat{a}_{1}^{*} \cdot \mu_{c}^{\prime} \cdot \mu_{\alpha}{ }^{3} \cdot \hat{a}_{3}\right)\left(\hat{k}_{3} \cdot \mu_{\alpha}{ }^{2} \cdot \hat{a}_{2}\right)-\frac{z e}{m_{\alpha} \omega_{3}}\right. \\
& \cdot\left(\hat{a}_{1}{ }^{*} \cdot \mu_{c}{ }^{\prime} \cdot \hat{a}_{3}\right)\left(\underline{k}_{3} \cdot \mu_{\alpha}{ }^{2} \cdot \hat{a}_{2}\right)+\frac{\left(\hat{a}_{1}{ }^{*} \cdot \mu_{\alpha}{ }^{3} \cdot \hat{a}_{3}\right)\left(\underline{k}_{2}-i \delta\right) \cdot \mu_{\alpha}{ }^{2} \cdot \hat{a}_{2}}{\left(\omega_{2}-\underline{k}_{2} \cdot \underline{v}_{\alpha 0}\right)} \\
& =\frac{1}{2} \sum_{\alpha} \frac{\mathrm{ze} \omega_{\alpha}^{2}\left(\mathrm{k}_{2}-\mathrm{i} \delta\right)}{\mathrm{m}_{\alpha}\left(\omega_{3}-\mathrm{k}_{3} \cdot \mathrm{v}_{\mathrm{ox}}\right)\left(\omega_{2}-\mathrm{k}_{2} \cdot \mathrm{v}_{\mathrm{ox}}\right) \Delta_{2}}\left[\left(1+\frac{\mathrm{k}_{2} \mathrm{v}_{\mathrm{ox}}}{\omega_{2}}\right)\left(\bar{\omega}_{2}-\mathrm{k}_{2} \mathrm{v}_{\mathrm{ox}}\right) \frac{\mathrm{b}_{2}}{\sqrt{1+\mathrm{b}_{2}{ }^{2}}}\right. \\
& \left.+\left(z \Omega_{0}+\frac{\left(\omega_{2}-\underline{k}_{2} \cdot \underline{v}_{0 x}\right) k_{2} v_{o y}}{\omega_{2}}\right) \frac{1}{\sqrt{1+b_{2}^{2}}}\right] \text {, }
\end{aligned}
$$


$\mathrm{v}_{321}$ is obtained by interchanging $\omega_{1}$ and $\omega_{3}$ in $\mathrm{v}_{123}$ and

$$
\begin{aligned}
& v_{231}=2 \pi i \sum_{\alpha} \frac{\rho_{\alpha 0} \mathrm{ze}}{\mathrm{m}_{\alpha}}\left[\left(\hat{\mathrm{a}}_{2}{ }^{*}+\frac{\hat{\mathrm{a}}_{2}^{*} \cdot \underline{\mathrm{v}}_{\alpha 0} \mathrm{k}_{2}}{\omega_{2}}\right) \cdot \mu_{c}{ }^{2}\left\{\frac{\underline{\mathrm{k}}_{3}}{\omega_{3}}\left(\hat{\mathrm{a}}_{3} \cdot \mu_{\alpha} \cdot \hat{\mathrm{a}}_{1}\right)+\frac{\underline{\mathrm{k}}_{1}}{\omega_{1}}\left(\hat{\mathrm{a}}_{1} \cdot \mu_{\alpha}{ }^{3} \cdot \hat{\mathrm{a}}_{3}\right)\right\}\right] \\
& =\frac{1}{2} \sum_{\alpha} \frac{\omega_{\alpha}^{2}}{\Delta_{2}} \frac{z_{e}}{m_{\alpha}}\left(\frac{\left(\bar{\omega}_{2}-k_{2} v_{o x}\right) b_{2}+\Omega_{o}}{\sqrt{1+b_{2}^{2}}}\right)\left[\frac{k_{3}}{\omega_{3}\left(\bar{\omega}_{1}-\underline{k}_{1} v_{o x}\right)}+\frac{k_{1}}{\omega_{1}\left(\bar{\omega}_{3}-\underline{k}_{3} v_{o x}\right)}\right]
\end{aligned}
$$

We consider $\omega, k, \Delta k$ real and $A_{3}$ is the pump of fixed amplitude. The small amplitudes are described by equations (5.2) - (5.4). We take A's to be space independent so that

$$
A_{1}=\left[\exp \left\{i \int_{0}^{t} \frac{1}{2} \Delta \omega d t\right\}\right] \psi^{*}
$$

and then we find

$$
\left[\frac{\partial^{2} \psi}{\partial t^{2}}+i \Delta \omega \frac{\partial \psi}{\partial t}+\frac{(\Delta \omega)^{2} \psi}{4}\right]=\nu_{\alpha}^{2} \psi,
$$

where

$$
\nu_{\alpha}^{2}=\left(v_{123} v_{213} A_{3}^{2}\right)
$$

Neglecting $i \Delta \omega \frac{\partial \psi}{\partial t}$, it follows that the solution of (5.9) by W.K.B. method assumes the form

$$
\psi=\frac{1}{\sqrt{g}}\left[A_{1} \exp \left\{i \int_{0}^{t} g(t) d t\right\}+B_{2} \exp \left\{-i \int_{0}^{t} g(t) d t\right\}\right],
$$

with

$$
g=\left\{v_{123} \quad v_{213} A_{3}^{2}-\frac{(\Delta \omega)^{2}}{4}\right\}^{\frac{1}{2}}
$$

When the wave packet drifts along $x$ the time -increment dt can be written as $\begin{aligned} d t=(d x) /\left(\frac{\partial \omega}{\partial k}\right) & \text { to obtain } x_{t} \\ & A_{1} \sim\left[\exp \int_{0}^{g(x) d x] /\left(\frac{\partial \omega}{\partial k}\right)},\right.\end{aligned}$

where the integration is limited to the instability zone. It is interesting to note that the threshold value of the wave can be calculated when $g<0$ 


$$
A_{3}=\frac{\Delta \omega}{2 \sqrt{v_{123} \mathrm{~V}_{213}}}
$$

Thus the value of the frequency shift can be obtained from (5.14) as

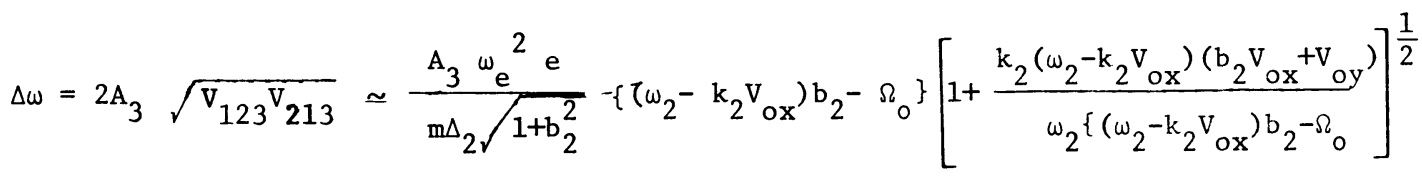

$$
\begin{aligned}
& \times\left[\frac{1}{\left(\omega_{3}-k_{3} v_{o x}\right)\left(\omega_{2}-k_{2} v_{o x}\right)}\left\{\frac{k_{3}}{\omega_{3}\left(\omega_{1}-k_{1} v_{o x}\right)}+\frac{k_{1}}{\omega_{1}\left(\omega_{3}-k_{3} v_{o x}\right)}\right]^{\frac{1}{2}}\right.
\end{aligned}
$$

where the assumption $\omega_{\mathrm{e}}^{2} / \mathrm{m} \gg \omega_{i}^{2}$ is invoked.

With the following numerical values

$$
\begin{aligned}
& \delta=0 \\
& \omega_{1}=\omega_{3}^{1}=2.7 \times 10^{15} \mathrm{sec}^{-1}=0 \\
& \omega_{2}=\omega_{e}=5.6 \times 10^{11} \mathrm{sec}^{-1} \\
& k_{3}-k_{2}=k_{1}=19 \mathrm{~cm}^{-1} \\
& a_{1}, a_{3} \simeq \frac{10^{6}}{3} \text { e. s. u } / \mathrm{cm} ;
\end{aligned}
$$

it turns out that $\Delta \omega \simeq 10^{8} \mathrm{sec}^{-1}$ which is in good agreementwith that of Montgomery [137

Similarly, considering the space dependence only, one can calculate the wave number shift from equations (5.2) - (5.4).

6. DISCUSSION.

The general features of density gradient and magnetic field in the nonlinear interactions of plasma oscillations have been investigated. This is important in connection with its use as an optical density probe suggested by $\operatorname{rrol1}$, Ron and 
Rostoker [13] or as a controlled source of plasma oscillations conceived by Montgomery [13] the frequency shift will deviate with the increased value of the density gradient. However, strong magnetic field will have only influence on the frequency shift.

ACKNOWLEDGMENT. This work was partially supported by a summer grant from the East Carolina University Research Committee. Authors express their sincere thanks to Mrs. Lela Skinner for typing the final manuscript.

\section{REFERENCES}

1. Liu, C. S. and Rosenbluth, M. N. Parametric decay of electromagnetic Waves into two plasmons and its consequences, Phys. Fluids. 19 (1976) 967-971.

2. Liu, C. S., Rosenbluth, M. N., and White, R. B. Raman and Brillouin scattering of electromagnetic waves in inhomogeneous plasmas, Phys. Fluids. 17 (1974) 1211-1219.

3. Nicholson, D. R. Parametric instabilities in plasma with sinusoidal density modulation, Phys. Fluids 19 (1976) 889-895.

4. Thomson, J. J. Finite-Band width Effects on the Parametric Instability on the Parametric Instability in an inhomogeneous Plasma, Nuclear Fusion 15 (1975) 237-247.

5. Nishikawa, K. Parametric Excitation of Coupled Waves, I. General Formulation, J. Phys. Soc. Japan 24 (1968) 918-922.

6. Gorbunov, L. M., Domrin, V. I., and Ramazashvili. Stimulated Raman scattering and the penetration of an electromagnetic wave into an inhomogeneous plasma, Sov. Phys. JETP 43 (1976) 1128-1138.

7. Ikemura, T. and Nishikawa, K. Parametric Coupling of Langmuir Waves in an inhomogeneous plasma, J. Phys. Soc. Japan, 32 (1972) 1368-1376.

8. Ikemura, T. Parametric Coupling of Two Langmuir Waves in an inhomogeneous plasma. II. Stabilizing Effects Due to the ponderomotive force created by an intense pump field, J. Phys. Soc. Japan 41 (1976) 281-291.

9. Galeev, A. A. and Sagdeev, R. Z. Parametric Phenomena in a plasma, Nucl. Fusion 13 (1973) 603-621. 
10. Perkins, F. W. and Flick, J. Parametric instabilities in inhomogeneous plasmas, Phys. Fluids 14 (1971) 2012-2018.

11. Eubank, H. P. Threshold electric field for excitation of parametric instabilities in inhomogeneous plasmas, Phys. Fluids 14 (1971) 2551-2552.

12. Krol1, N. M. Ron, A. and Rostoker, N. Optical Mixing as a plasma density probe, Phys. Rev. Lett. 13 (1964) 83-85.

13. Montgomery, D. On the resonant excitation of plasma oscillations with laser beams, Physica 31 (1965) 693-702. 


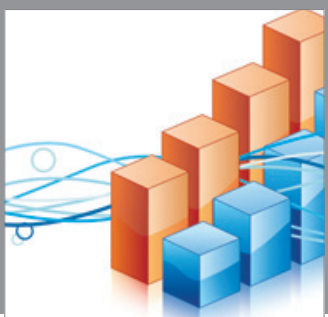

Advances in

Operations Research

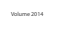

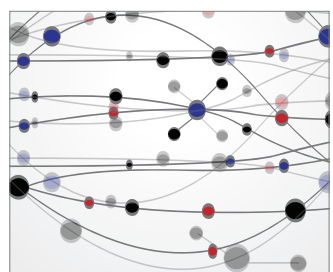

\section{The Scientific} World Journal
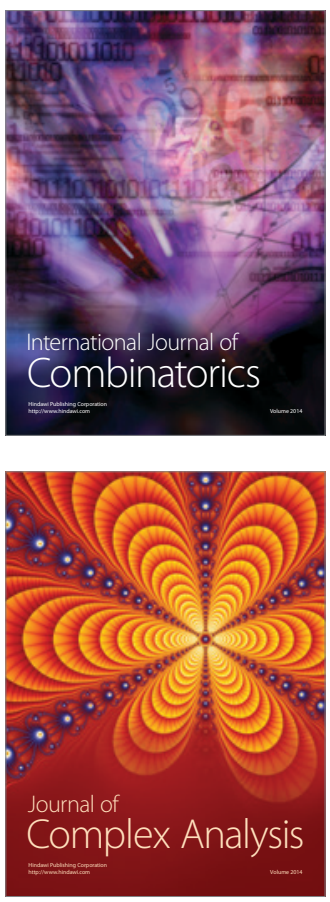

International Journal of

Mathematics and

Mathematical

Sciences
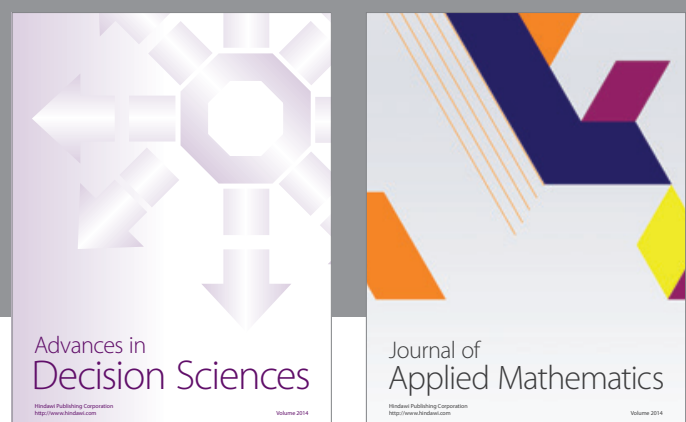

Journal of

Applied Mathematics
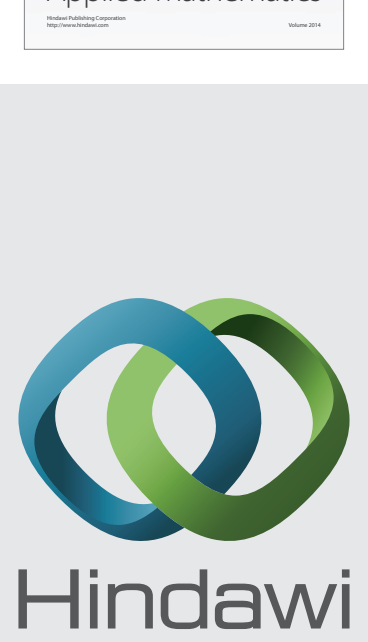

Submit your manuscripts at http://www.hindawi.com
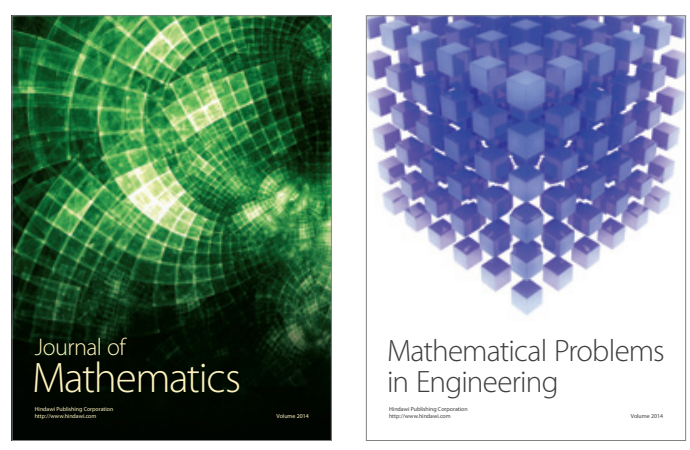

Mathematical Problems in Engineering
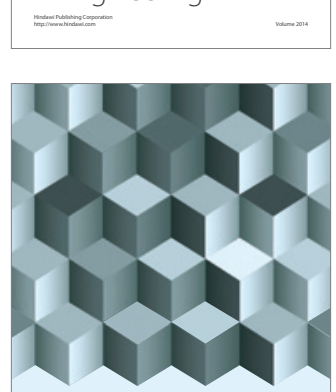

Journal of

Function Spaces
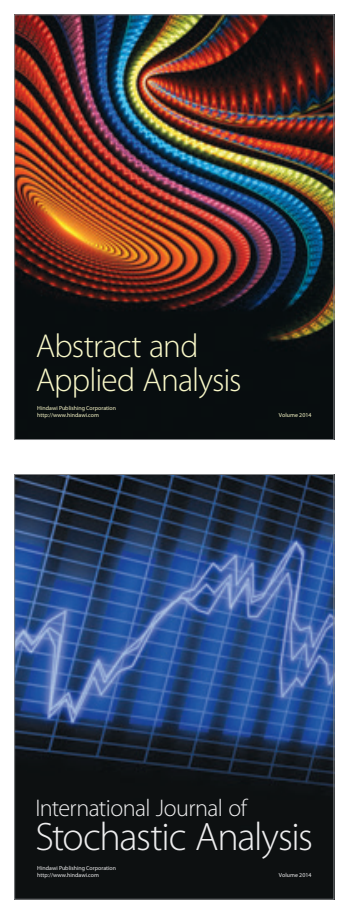

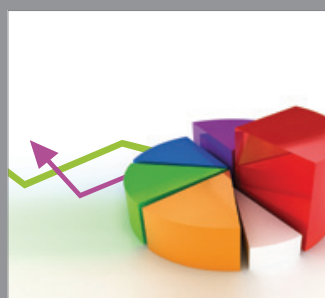

ournal of

Probability and Statistics

Promensencen
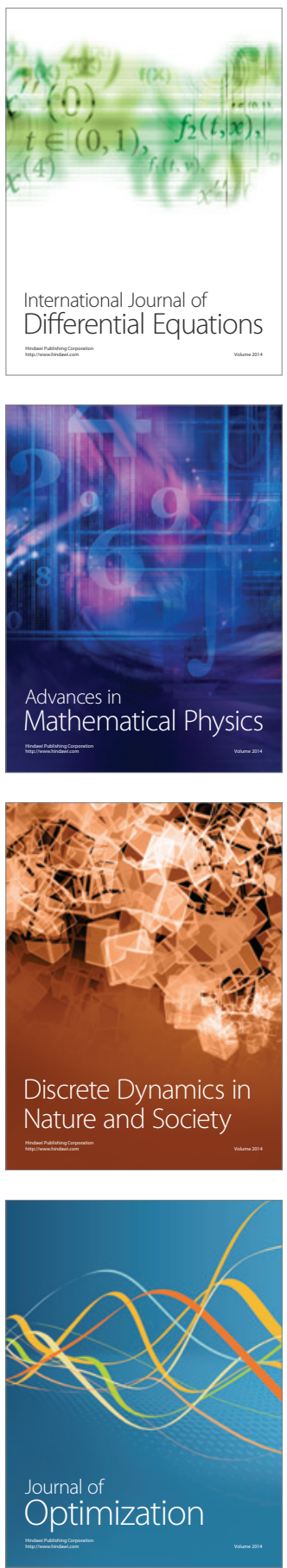